\title{
Clinical value and application of preoperative CT-guided hookwire localization of solitary pulmonary nodules for video-assisted thoracic surgery
}

\author{
Gen Yan ${ }^{\mathrm{a}}$, Xiaofang Cheng ${ }^{\mathrm{b}, \mathrm{c}}$, Shuohua $\mathrm{Wu}^{\mathrm{d}}$, Yuxi Ge ${ }^{\mathrm{e}}$, Shanhua $\mathrm{Li}^{\mathrm{f}}$ and Yinghua Xuan ${ }^{\mathrm{g}, *}$ \\ ${ }^{a}$ Department of Radiology, The Second Affiliated Hospital of Xiamen Medical College, Xiamen, Fujian, \\ China \\ ${ }^{\mathrm{b}}$ The First Affiliated Hospital of Jinan University, Guangzhou, Guangdong, China \\ ${ }^{\mathrm{c}}$ Department of Radiology, Affiliated Brain Hospital of Guangzhou Medical University, Guangzhou \\ Huiai Hospital, Guangzhou, Guangdong, China \\ ${ }^{\mathrm{d}}$ Department of Medical Imaging, The Second Affiliated Hospital of Shantou University Medical College, \\ Shantou, Guangdong, China \\ ${ }^{\mathrm{e}}$ Department of Radiology, Affiliated Hospital, Jiangnan University, Wuxi, Jiangsu, China \\ ${ }^{\mathrm{f}}$ Department of Basic Medical Sciences, School of Medicine, Xiamen University, Xiamen, Fujian, China \\ ${ }^{\mathrm{g}}$ Department of Basic Medicine, Xiamen Medical College, Xiamen, Fujian, China
}

\begin{abstract}
.
BACKGROUND: Video-assisted thoracic surgery (VATS) is a minimally invasive technique for the diagnosis and management of small pulmonary nodular lesions However, the identification of some lung nodules remains difficult.

OBJECTIVE: This research aimed to investigate the clinical value of preoperative computed tomography (CT)-guided hookwire localization of solitary pulmonary nodules (SPNs) for thoracoscopic resection.

METHODS: Seventy-one patients with 74 SPNs underwent VATS wedge resection after CT-guided hookwire localization. The mean diameter of the SPNs was $8.50 \pm 4.53 \mathrm{~mm}$,,besides, the mean distance from the SPNs to the parietal pleura was $16.81 \pm$ $5.23 \mathrm{~mm}$.

RESULTS: Sixty-nine of the 74 nodules were successfully localized using a CT-guided hookwire. The success rate of CT-guided localization was $93.2 \%$. The average localization time was $15.23 \pm 7.21 \mathrm{~min}$ per lesion. Seven patients $(9.5 \%)$ had asymptomatic pneumothorax and $10(13.5 \%)$ had minimal needle tract parenchymal hemorrhages after localization no clinical intervention was required for these patients. The rate of success for VATS wedge resection of the SPNs was $100 \%$. Histological analysis of the SPNs revealed malignant disease in $67.4 \%$ of the patients.

CONCLUSIONS: Preoperative CT-guided hookwire localization for thoracoscopic resection is a safe and effective operation for the identification and stable fixation of SPNs.
\end{abstract}

Keywords: Solitary pulmonary nodules, CT-guided, hookwire, video-assisted thoracic surgery

\footnotetext{
${ }^{*}$ Corresponding author: Yinghua Xuan, Department of Basic Medicine, Xiamen Medical College, Xiamen, Fujian 361008, China. Tel.: +86 18112385686; E-mail: yhxuan@jiangnan.edu.cn.
}

0928-7329 (c) 2022 - The authors. Published by IOS Press. This is an Open Access article distributed under the terms of the Creative Commons Attribution-NonCommercial License (CC BY-NC 4.0). 


\section{Introduction}

Solitary pulmonary nodules (SPNs) are defined as solitary, well-circumscribed lesions with increased opacity in the lung that measure less than $3 \mathrm{~cm}$ in diameter [1,2], with no associated lymphadenopathy, atelectasis, pneumonia, or pleural effusion [3]. With the growing popularity and efficacy of computed tomography $(\mathrm{CT})$, the detection rate of solitary pulmonary nodules has been increasing. It has been reported that approximately 150,000 SPNs are detected annually in the United States [4], and they are often detected incidentally during examination for another reason. According to a study by Li et al. [6], more than 50\% of resected pulmonary nodular lesions (PNLs) are malignant. Because of this high incidence rate, there is a need for rapid and definite pathological diagnosis of PNLs [6]. Videoassisted thoracic surgery (VATS) causes minimal lung volume loss and little postoperative incidence rate and death rate [7] (Fig. 1a-f). On the basis of multivariate logistic regression analytical method, acupuncture frequency was the only independent risk factor for location-dependent pneumothorax. And good surgical technique mitigates risks as far as possible [8]. Therefore, it is one of the standard minimally invasive techniques used for the diagnosis and management of small PNLs. VATS is a type of thoracic surgery that provides excellent visualization despite limited access to the thorax. It is mainly applied to the diagnosis and management of pulmonary, mediastinal, and pleural lesions. Because there is no formal thoracotomy incision (which requires placement of a retractor with rib distraction), VATS leads to significantly reduced postoperative pain and earlier recovery to normal function [9]. In patients with an underlying malignancy, VATS should be performed to verify the indication for chemotherapy and to complete the staging process [12].

A primary factor that limits the success rate of thoracoscopic resection of subcentimeter or lowattenuation pulmonary nodules via VATS is the accurate localization of the target nodule as it is difficult to distinguish or palpate thesenodules intraoperatively [13]. Moreover, a majority of small nodules that are deeply situated in the lung, or have ground glass opacities, can be difficult to localize with VATS. Inappropriate nodule localization might lead to a prolonged surgical duration because of the time taken to search for the nodule or conversion to an unplanned open thoracotomy [8]. An up to 46\% thoracotomy conversion rate was reported in the research which is contributed to Suzuki et al. [10] because of the inability to visualize or palpate the PNLs. In such situations, preoperative marking prior to thoracoscopy to facilitate the localization procedure is useful [6]. Since the development of radiologic localization techniques, the rate of successful VATS for resection of pulmonary nodules has improved, and the failure rate has decreased from 45 to $4 \%$ [15].

The techniques proposed for identifying target PNLs are as follows: 1) noninvasive intraoperative methods using pressure sensors orultrasonography 2) inserting percutaneous localizers, such as hookwires or contrast media, and 3) inserting a localizer transbronchially [12]. Each kind of these localization methods have certain advantages and limitations. Various techniques have been described for the percutaneous localization of SPNs, including CT-guided dye injection, intraoperative ultrasound localization, electromagnetic navigation bronchoscopy, and CT-guided hookwire localization. A study by Kleedehn et al. [18] found that the CT-guided dye method resulted in more pain, which occurred because of pleural irritation caused by the dye. Another disadvantage is that the depth of the intraparenchymally situated lesion cannot be exactly determined during VATS, and vision might be affected by the dispersed or leaking dye intraoperatively [19]. Intraoperative ultrasound localization and electromagnetic navigation bronchoscopy both require specialized equipment and training. Moreover, the intraoperative ultrasound localization technique requires complete collapse of the lung, which is often impossible in patients with emphysema [8]. CT-guided hookwire marking was demonstrated to yield high technical and clinical 
A
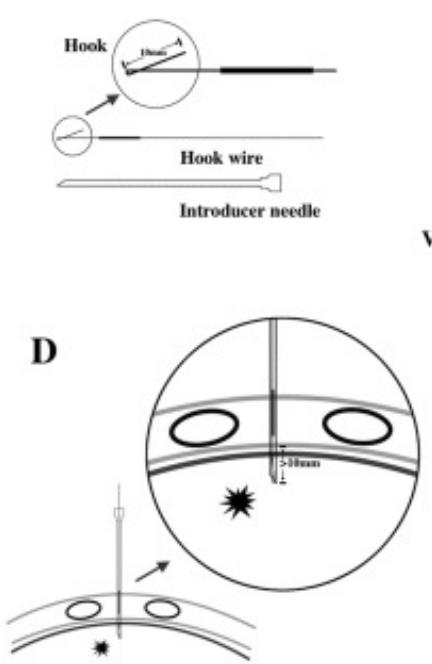

B

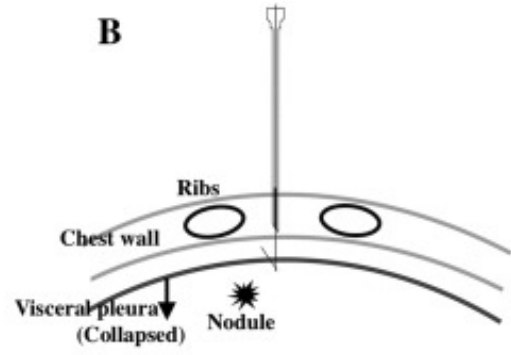

$\mathbf{E}$

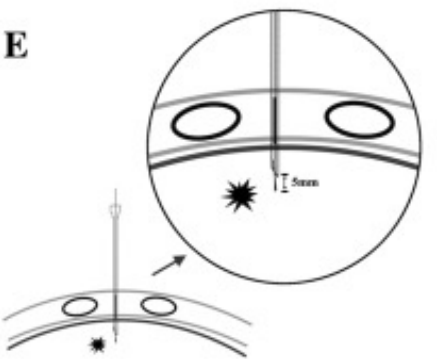

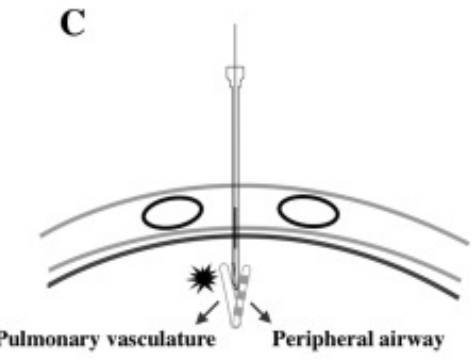

F

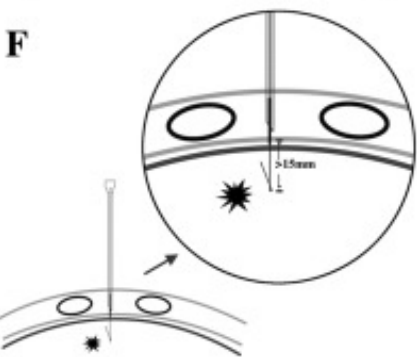

Fig. 1. Technical diagram of the CT-guided hook wire positioning technology. (A): Hook wire equipment. (B): Hook wire should not be inserted too shallow, otherwise it is easy to pull out the hook wire when the lung collapses. (C): The hook wire should also not be inserted too deeply; otherwise the possibility of pulmonary vascular or airway damage will increase. (D), (E) and (F): Three-step approach to insert the hook wire and take out the guide needle. (D): Hook wire and guide needle should be inserted no less than $10 \mathrm{~mm}$ below the visceral pleural surface. (E): The insertion distance of hook wire should be $5 \mathrm{~mm}$ above the tip of guide wire. (F): Guide needle could be unreeved entirely.

success rates (93.6-97.6\%) [18]. A retrospective study that included a large sample size ( $>100$ cases) [8] on the application of preoperative CT-guided hookwire positioning of SPNs over 5 years showed the acceptable safety and reliability of this technique. Most physicians favor CT-guided hookwire localization because it has several major outstanding advantages over the other methods used for lesions localization, such as shorter procedure times and the capability to pull the fixed tissue superficially, thereby facilitating precise wedge resection [11].

In this study, we aimed to investigate the clinical value of preoperative CT-guided hookwire localization of SPNs for thoracoscopic resection with regard to security, dependability, and convenience of the technology using our experience and clinical results with this technology [8].

\section{Patients and methods}

This study was conducted in accordance with the principles of the Declaration of Helsinki. The Institutional Review Board of the Affiliated Hospital of Jiangnan University approved the study protocol, and written informed consent was obtained from all patients after providing detailed explanations. Seventyone patients with 74 solitary pulmonary nodules underwent VATS wedge resection after preoperative CT-guided hookwire localization in our hospital between September 2014 and May 2016. The patients included 41 women and30 men (mean age: $60.3 \pm 7.9$ years old; age range: $33-83$ ). The operating surgeon and radiologists conjointly reviewed the available CT scans of all cases to determine the viability of hookwire nodule localization and VATS resection and establish a treatment scheme. The inclusion criteria were as follows: (1) diameter of the lesion $\leqslant 30 \mathrm{~mm}$ (2) SPNs without invasion into the viscera pleura (3) total number of SPNs $\leqslant 3$ and (4) no enlarged hilar or mediastinal lymph nodes. Sixty-eight 


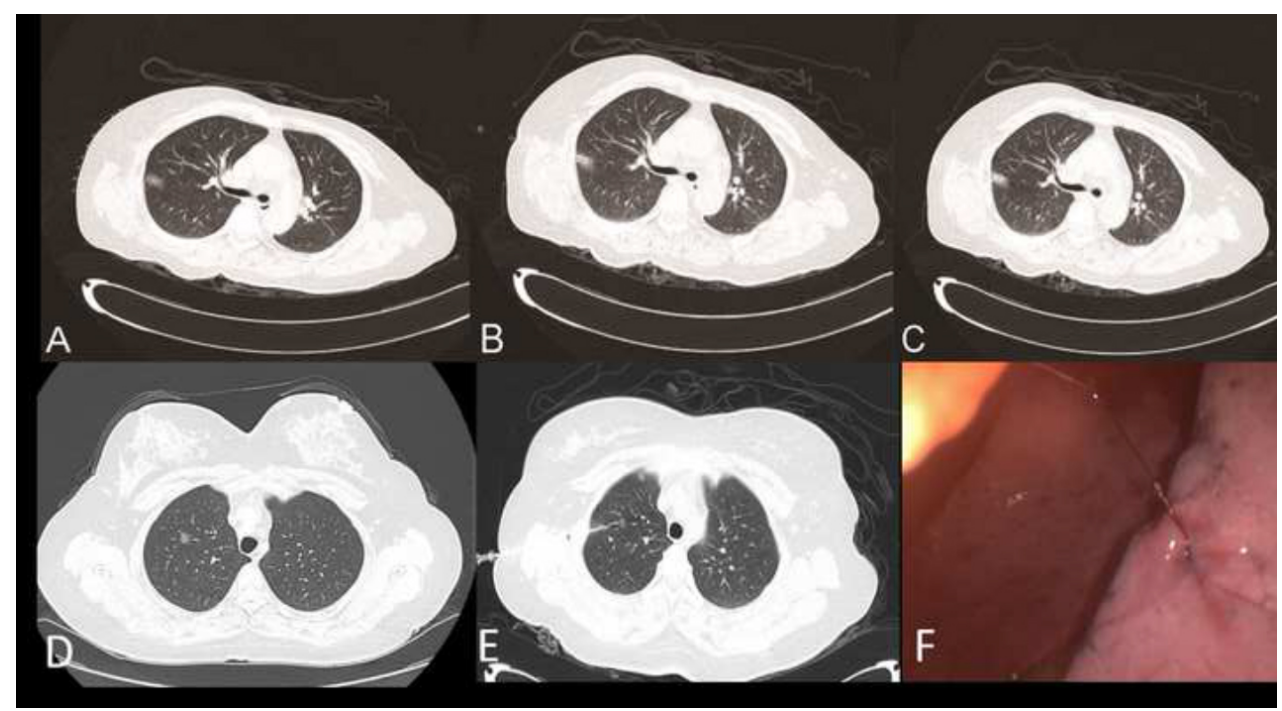

Fig. 2. (A, B) Computed tomography scan before and after marking the pulmonary nodule using the hookwire of Case 1 . (C) Anchorage of the hookwire in the lung parenchyma in Case 1. (D-E) Computed tomography scan before and after marking the pulmonary nodule using the hookwire in Case 2. (F) Lesion under VATS in Case 2. VATS, video-assisted thoracic surgery.

patients had solitary lesions and three patients had two separate lesions (74 lesions). Of the 74 nodules, 43 were located in the right lung and 28 in the left lung. The maximum diameter of the SPNs ranged from 3.97 to $13.03 \mathrm{~mm}$ (average: $8.50 \pm 4.53$ ), and the depth of the lesion with relative to the nearest pleural surface ranged from 11.58 to $22.04 \mathrm{~mm}$ (average: $16.81 \pm 5.23$ ). There were 12 patients with a history of other malignant lesions (three with cervical cancer, three with breast cancer, two with renal cancer, and four with colon cancer). The SPNs in the remaining 62 patients were detected during physical examinations or incidentally.

All patients were intramuscularly injected with pethidine and were placed on the CT scanner (Siemens, Emotion 64) in a position that allowed the shortest direct access path for hookwire insertion [8]. A localized CT scan was performed to plan the access route of the needle and to measure the depth of the nodule from the external surface of the chest wall. After cleaning and sterilizing the skin around the planned needle puncture site, local anesthesia was administered using $2 \%$ lidocaine. A mammographic hookwire system (breast localization needle, $20 \mathrm{G} \times 100 \mathrm{~mm}$, Pajunk Medical Co., Germany) was used for localization. The hookwire was delivered around the nodule, anchoring the system into the lung. A second CT scan was performed to confirm if the trocar tip was located in the lesion, and if it was, the trocar was pushed forward again by $5 \mathrm{~mm}$. Then, the hooked wire was released, and the trocar was withdrawn, then another CT scan was performed. The front of the wire was unfolded into a hook-like shape, and the tip was advanced beyond the lesion by 3-5 mm (Fig. 2a-f). The hookwire extending outside the chest wall was bent and loosely covered with sterile gauze adequately. All patients were then transferred to the operating room directly after localization. The interval time between preoperative localization and VATS wedge resection was within one hour.

\section{Results}

The mean duration of the preoperative localization procedure was $15.23 \pm 7.21$ min per lesion. Among the 74 nodules, 69 were successfully localized using CT-guided hookwire without any major 
Table 1

Definitive diagnosis of the solitary pulmonary nodules

\begin{tabular}{lc} 
Primary lung cancer & \\
Adenocarcinoma & $37(50 \%)$ \\
Metastatic lung cancer & $12(16 \%)$ \\
Inflammatory pseudotumor & $13(17.6 \%)$ \\
Tuberculoma & $4(5.4 \%)$ \\
Hamartoma & $2(2.7 \%)$ \\
Atypical adenomatous hyperplasia & $2(2.7 \%)$ \\
Lymph node hyperplasia & $2(2.7 \%)$ \\
Sclerosing hemangioma & $1(1.4 \%)$ \\
Mucosa-associated lymphoma & $1(1.4 \%)$ \\
\hline
\end{tabular}

Table 2

\begin{tabular}{lc} 
Origin of primary malignancy \\
\hline Lung & 1 \\
Cervix uterus & 3 \\
Breast & 4 \\
Osteosarcoma & 1 \\
Colon & 3 \\
\hline
\end{tabular}

postoperative complications, and the success rate was 93.2\%. Two cases of intraoperative hookwire slippage and three of hookwire dislodgement occurred. Founded on the puncture site of the lung surface and the lung hematoma caused by the puncture, an extended resection was required in five patients, and the SPN was excised using thoracoscopy. Complications related to the wire localization technique included minimal and asymptomatic pneumothorax (7 cases, 9.5\%) and minimal needle tract parenchymal hemorrhages (10 cases, 13.5\%).No clinical intervention was required in these cases. There were no serious postoperative complications, like hemothorax or air embolism, in any patient. All patients had an uncomplicated postoperative course, and the mean duration of postoperative hospitalizationstay was around 7 days.

Postoperative pathological examination showed that out of 74 SPNs, there were 37 cases of primary lung adenocarcinoma, 12 of metastatic lung cancer, 13 of inflammatory pseudotumor, 4 of tuberculoma, 2 of hamartoma, 2 of atypical adenomatous hyperplasia, 1 of sclerosing hemangioma, 2 of lymph node hyperplasia, and 1 of mucosa-associated lymphoma (Table 1). Overall, malignant disease accounted for $67.4 \%$ of all cases, and the origins of primary malignancy are summarized in Table 2.

\section{Discussion}

In this pilot study, the CT-guided hookwire localization method was used for 74 SPNs in 71 patients. Localization was successfully performed for 69 SPNs (successful implantation rate of 93.2\%). This is consistent with the findings of some anterior studies that had a observably high success rate of 93.6-97.6\% [19] and confirmed the superiority of CT-guided hook wire positioning for SPNs before operation compared to a $48 \%$ success rate without guidance [20]. The system used was developed for the localization of breast nodules. According to the study by Wicky et al. [22], this mammographic hookwire system has characteristics that offer an efficient anchorage and allows nodules as deep as $30 \mathrm{~mm}$ below the pleura to be located identified and resected. The depth of the lesion with relative to the nearest pleural surface in our study was 3.97 to $13.03 \mathrm{~mm}$ (average: $16.81 \pm 5.23$ ). The average localization time was $15.23 \pm 7.21 \mathrm{~min}$ per lesion This was slightly shorter than that reported in earlier studies $[6,21]$. 
Following the protocol described in the study by Ciriaco et al. [23], the dislodgement rate was reduced by positioning the patients on the operating table adequately and retaining a suitable length of the hookwire outside the chest. In addition, the hook at the distal end of the wire ensured more safe and reliable implantation in the pulmonary nodules. Out of the 74 nodules evaluated in our study, there were two cases of wire slippage and three of hookwire displacement. The dislodgement rate $(6.8 \%)$ was acceptable and comparable to that reported by Ciriaco et al. (7.5\%). Dislodgement occurred mostly during the VATS procedure (when the lung was collapsed, which modified the position) rather than during puncture (when the lung was inflated). The probability of slippage was related to the distance between the hookwire and the pleura and the flexibility of the lung tissue itself. Among the dislodgement cases, there were three cases of superficial pulmonary nodules, and the hookwire was placed too close to the surface. The flexibility of the pulmonary tissues in two patients with a long-term history of smoking was poor, and intraoperative pulling may have led to slippage of the hookwire. We believe that if the hookwire had been placed deeper, the likelihood of slippage would have been reduced. If the distance from the SPN to the lung surface was more than $2 \mathrm{~cm}$, the trocar was pushed forward by $5 \mathrm{~mm}$ after the puncture needle passed through the lesion. If the distance from the SPN to the lung surface was less than $2 \mathrm{~cm}$, the puncture needle was pushed forward by more than $2 \mathrm{~cm}$ from the lung surface to prevent slippage of the hookwire. Of the five cases of slippage and displacement of the localization needle in our research, the physical distance from the puncture needle to the lung surface was less than $2 \mathrm{~cm}$ in four cases. In addition, according to the experience of Hanauer et al. [24], maintaining a suitable length of wire cable outside the thorax can reduce the dislodgement rate, as it allows the wire to follow the collapsed lung during selective pneumothorax or ventilation. During VAT wedge resection, the localization needle had to be lifted to prevent the endoscopic gastrointestinal anastomosis (endo-GIA) device from cutting to the localization needle, but the lifting force could not be too great to prevent slippage of the localization needle.

Some common postoperative complications, like pneumothorax and pulmonary hemorrhage, may occur during localization of the hookwire. It was reported in a previous study that asymptomatic pneumothorax occurred in 7.5-49\%, pulmonary bleeding in 14.9-29.8\%, and hemorrhage into the pleural cavity in 0.6$3.5 \%$ of the cases [21]. Our study included seven cases $(9.5 \%)$ of asymptomatic minimal pneumothorax that did not require any treatment. Of these, five cases were caused by multiple punctures, and in two cases, the patient had a history of chronic obstructive pulmonary disease. The incidence of pulmonary hemorrhage was $13.5 \%(10 / 74)$ an extended resection was required in five of these patients. We believe that a high skill level of the surgeon and a reduced number of punctures could effectively decrease the incidence of complications, like pneumothorax and alveolar hemorrhage. Pulmonary venous air embolism is a rare however important and serious complication during pulmonary nodule hookwire localization that could lead to a lethal outcome. Several cases of air embolism during hookwire marking have occasionally been reported in recent years [19]. However, this complication was not observed in our study. As suggested in a previous study, a short trajectory for the hookwire and the absence of prolonged lung inflation can decrease the possibility of air embolism [23].

Histological analysis of the SPN revealed malignant disease in $67.4 \%$ of the patients. Our work supports the anteriorly reported results in this field [28]. The data analysis highlights the fact that it is necessary to actively deal with nodules [29].

On the basis of our experience and the data from earlier studies [30], we concluded that the hookwire localization technique has the following advantages: (1) the procedure simplifies the surgery, with a high success rate of localization (2) the anchorage in the lung parenchyma is efficient, and generally causes no serious complications (3) during VATS, the nodules can be pulled to a superficial position with the 
long metal wire attached to the hookwire for wedge resection, which is beneficial for obtaining a rapid and accurate margin (4) nodules can be identified more quickly for frozen section examinations with the guidance of hookwire localization, thereby significantly shortening the intraoperative wait time [6]. However, because of the small sample size, our results should be interpreted with caution and need to be replicated and confirmed by further research with a larger sample size.

\section{Conclusion}

In summary, the main merits of preoperative CT-guided hookwire localization of pulmonary nodular lesions before VATS include technical ease, minor complications, shorter duration of surgery, and safe and effective fixation of the nodule allowing complete resection. Given all of these advantages, we highly recommended a preoperative CT-guided hookwire marking technique to localize solitary pulmonary nodules for thoracoscopic resection.

\section{Acknowledgments}

The authors are truly grateful to Fanghui Qin from the Department of Radiology of the Affiliated Hospital of Jiangnan University for his assistance with the data collection.

\section{Availability of data}

All the data and materials are available from the corresponding author upon reasonable request.

\section{Conflict of interest}

None of the authors have any conflict of interest to report.

\section{Funding}

This study was supported by the Science and Technology Program of Fujian Province of China (No. 2017D017); the Joint Funds for the Health and Education of Fujian Province, China (No. 2019-WJ-31); and the Institute of Respiratory Diseases, Xiamen Medical College (No. HXJB-06, 15).

\section{References}

[1] Shersher DD, Kim AW. Solitary pulmonary nodules Common surgical diseases. Springer, New York. 2015.

[2] Viggiano RW, Swensen SJ, Rosenow EC. Evaluation and management of solitary and multiple pulmonary nodules. Clin Chest Med. 1992; 3(13): 83-95.

[3] Ost D, Fein AM, Feinsilver SH. The solitary pulmonary nodule. N Engl J Med Clinical Practice. 2003; 348: 2535-2542.

[4] Erasmus JJ, Connolly JE, McAdams HP, Roggli VL. Solitary pulmonary nodules: Part I. Morphologic evaluation for differentiation of benign and malignant lesions. Radio Graphics. 2000; 20: 43-58. 
[5] Mosmann MP, Borba MA, Macedo FPNd, Liguori AdAL, Villarim Neto A, Lima KCd. Solitary pulmonary nodule and 18F-FDG PET/CT. Part 1: Epidemiology, morphological evaluation and cancer probability. Radiol Bras. 2016; 49: 35-42. doi: 10.1590/0100-3984.2014.0012.

[6] Li WT, Wang Y, He XH, Li G, Wang S, Xu L, Yuan Z. Combination of CT-guided hookwire localization and videoassisted thoracoscopic surgery for pulmonary nodular lesions: analysis of 103 patients. Oncol Lett. 2012; 4: 824-828. doi: 10.3892/ol.2012.800.

[7] Soardi GA, Perandini S, Motton M, Montemezzi S. Assessing probability of malignancy in solid solitary pulmonary nodules with a new Bayesian calculator: improving diagnostic accuracy by means of expanded and updated features. Eur Rad. 2015; 25: 155-162.

[8] Yao F, Wang J, Yao J, Xu L, Wang J, Gao L. Reevaluation of the efficacy of preoperative computed tomography-guided hook wire localization: A retrospective analysis. Int J Surg. 2018; 51: 24-30.

[9] Seo JM, Lee HY, Kim HK, Choi YS, Kim J, Shim YM, Lee KS. Factors determining successful computed tomographyguided localization of lung nodules. J Thorac Cardiovasc Surg. 2012; 143: 809-814.

[10] Suzuki K, Nagai K, Yoshida J, Ohmatsu H, Takahashi K, Nishimura M, Nishiwaki Y. Video-assisted thoracoscopic surgery for small indeterminate pulmonary nodules: indications for preoperative marking. Chest. 1999; 115: 563-568.

[11] Ichinose J, Kohno T, Fujimori S, Harano T, Suzuki S. Efficacy and complications of computed tomography-guided hook wire localization. Ann Thorac Surg. 2013; 96: 1203-1208.

[12] Chen YR, Yeow KM, Lee JY, Su IH, Chu SY, Lee CH, Cheung YC, Liu HP. CT-guided hook wire localization of subpleural lung lesions for video-assisted thoracoscopic surgery (VATS). J Formos Med Assoc. 2007; 106: 911-918.

[13] Kakkar B, Agarwal M, Sehgal A, Govil NP. Anesthetic considerations for video-assisted thoracoscopic surgery in a child with Glenn shunt for thoracic duct ligation and pleurodesis. Saudi J Anaesth. 2018; 12: 131-133.

[14] Kastl S, Langwieler TE, Krupski-Berdien G, Demir E, Izbicki JR. Percutaneous localization of pulmonary nodules prior to thoracoscopic surgery by CT-guided hook-wire. Anticancer Res. 2016; 26: 3123-3126.

[15] Watanabe K, Nomori H, Ohtsuka T, Kaji M, Naruke T, Suemasu K. Usefulness and complications of computed tomography-guided lipiodol marking for fluoroscopy-assisted thoracoscopic resection of small pulmonary nodules: experience with 174 nodules. J Thorac Cardiovasc Surg. 2006.

[16] Klinkenberg T, Dinjens L, Rienhart W, van der Wekken AJ, Groen HJ, Timens W, Van De Wauwer C, de Bock GH, Mariani M, van Geffen W. The efficacy and safety of CT-guided percutaneous hookwire localization in VATS for pulmonary nodules. Eur Respir J. 2016; 48: PA2821.

[17] Kleedehn M, Kim DH, Lee FT, Lubner MG, Robbins JB, Ziemlewicz TJ, Hinshaw JL. Preoperative pulmonary nodule localization: A comparison of methylene blue and hookwire techniques. AJR Am J Roentgenol. 2016; 207: 1334-1339.

[18] Ko KH, Huang TW, Lee SC, Chang W, Gao H, Hsu H. A simple and efficient method to perform preoperative pulmonary nodule localization: CT-guided patent blue dye injection. Clin Imaging. 2019; 58: 74-79.

[19] Suzuki K, Shimohira M, Hashizume T, Ozawa Y, Sobue R, Mimura M, Mori Y, Ijima H, Watanabe K, Yano M, Yoshioka $\mathrm{H}$, Shibamoto Y. Usefulness of CT-guided hookwire marking before video-assisted thoracoscopic surgery for small pulmonary lesions. J Med Imaging Radiat Oncol. 2014; 58: 657-662.

[20] Finley RJ, Mayo JR, Grant K, Clifton JC, English J, Leo J, Lam S. Preoperative computed tomography-guided microcoil localization of small peripheral pulmonary nodules: A prospective randomized controlled trial. J Thorac Cardiovasc Surg. 2015; 149: 26-31.

[21] Klinkenberg TJ, Dinjens L, Wolf RFE, van der Wekken AJ, van de Wauwer C, de Bock GH, Timens W, Mariani MA, Groen HJM. CT-guided percutaneous hookwire localization increases the efficacy and safety of VATS for pulmonary nodules. J Surg Oncol. 2017; 115: 898-904.

[22] Wicky S, Dusmet M, Doenz F, Ris HB, Schnyder P, Portier F. Computed tomography-guided localization of small lung nodules before video-assisted resection: experience with an efficient hook-wire system. J Thorac Cardiovasc Surg. 2002; 124: 401-403.

[23] Ciriaco P, Negri G, Puglisi A, Nicoletti R, Del Maschio A, Zannini P. Video-assisted thoracoscopic surgery for pulmonary nodules: rationale for preoperative computed tomography-guided hookwire localization. Eur J Cardio Thorac Surg. 2004; 25: 429-433.

[24] Hanauer M, Perentes JY, Krueger T, Ris HB, Bize P, Schmidt S, Gonzalez M. "Pre-operative localization of solitary pulmonary nodules with computed tomography-guided hook wire: report of 181 patients". J Cardiothorac Surg. 2016; 11: 5.

[25] Miyoshi K, Toyooka S, Gobara H, Oto T, Mimura H, Sano Y, Kanazawa S, Date H. Clinical outcomes of short hook wire and suture marking system in thoracoscopic resection for pulmonary nodules. Eur J Cardio Thorac Surg. 2009; 36: 378-382.

[26] Iguchi T, Yoshioka T, Muro M, Miyasho K, Inoue D, Hiraki T, Kanazawa S. Systemic air embolism during preoperative pulmonary marking with a short hook wire and suture system under CT fluoroscopy guidance. Jpn J Radiol. 2009; 27: 385-388. 
[27] Ramos AO, de Miguel Díez J, Maestu LP, de la Torre Fernández J. Arterial gas embolism: A rare complication of core needle biopsy in the diagnosis of solitary pulmonary nodule. Arch Bronconeumol. 2019; 55(9): 492-493.

[28] Hirai S, Hamanaka Y, Mitsui N, Morifuji K, Uegami S. Role of video-assisted thoracic surgery for the diagnosis of indeterminate pulmonary nodule. Ann Thorac Cardiovasc Surg. 2006; 12: 388-392.

[29] Chen S, Zhou J, Zhang J, Hu H, Luo X, Zhang Y, Chen H. Video-assisted thoracoscopic solitary pulmonary nodule resection after CT-guided hookwire localization: 43 cases report and literature review. Surg Endosc. 2011; $25: 1729$.

[30] Diederich S, Das M. Solitary pulmonary nodule: detection and management. Cancer Imaging 6(Special Issue a). 2006; S42-S46. 